\title{
APPROCHE EXPÉRIMENTALE POUR LA COMPRÉHENSION DES FOURS CULINAIRES DU HAUT MOYEN ÂGE : BILAN CRITIQUE ET PERSPECTIVES
}

\author{
Gaëlle BRULEY-CHABOT et Nicolas WARMÉ
}

L'étude morphologique et spatiale des fours culinaires n'est certes pas définitivement achevée, mais les jalons principaux ont été explorés et posés (BRULEY-СНАBOT 2007). L'aspect expérimental fait partie de notre base de réflexion depuis de nombreuses années; il convient désormais de transformer nos tentatives ponctuelles en pratiques expérimentales plus rigoureuses, afin de voir si cela peut répondre à certaines de nos interrogations quant à l'utilisation de ces fours.

Après un bilan des questions soulevées par l'archéologie, nous présenterons les diverses tentatives de cuissons réalisées par nos soins depuis 1996, leurs résultats et les problèmes qu'ils soulèvent et enfin nos perspectives et projets.

\section{LES QUESTIONS POSÉES PAR L'ARCHÉOLOGIE}

\section{LA RUBÉFACTION COMME CRITÈRE DE COMPARAISON}

Ces fours majoritairement creusés sont une structure récurrente des villages du haut Moyen Âge, au même titre, notamment, que les fonds de cabane et silos. Leur élaboration est simple et leur morphologie rappelle celle des fours à pain traditionnel. La cavité hémisphérique creusée sert à la fois de chambre de chauffe et de cuisson. Le four est associé à une fosse de travail qui est mise en œuvre en amont afin d'employer l'une des parois comme base de creusement pour le four (fig. 1).

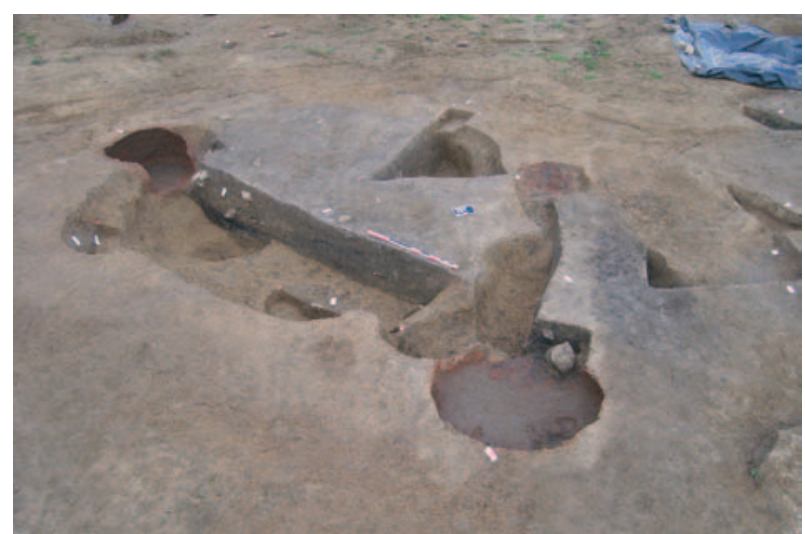

Le critère le plus pertinent et le plus visible d'une cuisson est la rubéfaction. Elle est observable à la fois à la fouille et lors d'expérimentations, en perforant les croûtes des soles et des parois (fig. 2). Les coupes ainsi réalisées permettent de noter les épaisseurs, la coloration et la dureté des couches rubéfiées.

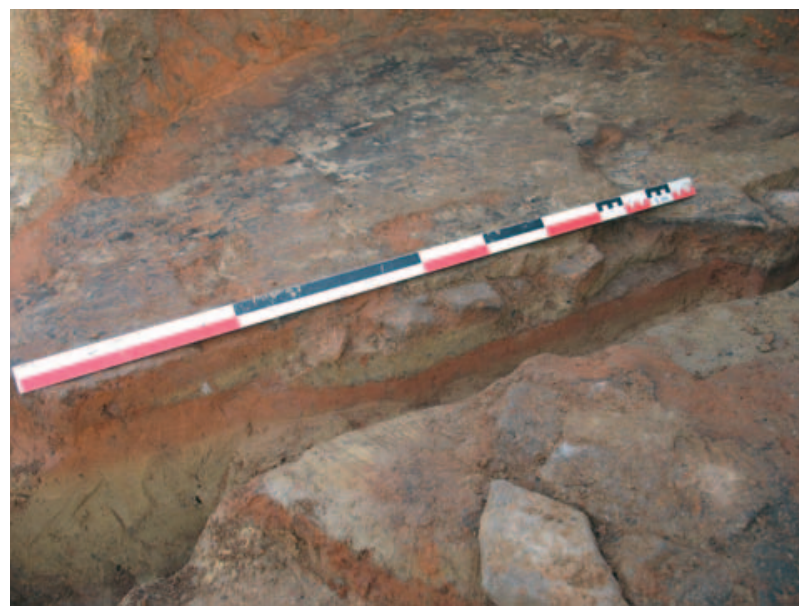

Fig. 2 - Vues de soles en coupe photo et relevé.

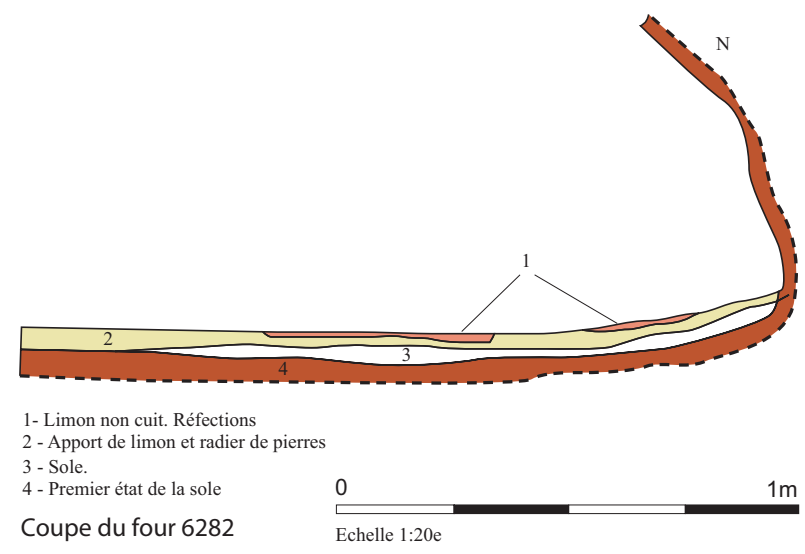

La rubéfaction est la résultante de plusieurs facteurs parmi lesquels : la durée d'exposition au feu, la température du foyer, la récurrence des chauffes mais aussi la morphologie et la taille de la chambre de cuisson. L'individualisation de chacun reste impossible car il semble que ce soit la

Fig. 1 - Vue d'un ensemble de fours.

Approche expérimentale pour la compréhension des fours culinaires du haut Moyen Âge : bilan critique et perspectives. 
combinaison de ces différents facteurs qui détermine si la structure est « bien cuite » ou « peu cuite. Ces interactions sont donc multiples et peuvent évoluer d'une chauffe à l'autre. Nous n'en avons, alors, qu'un témoignage " cumulé ». Le facteur " taille de la chambre de cuisson » semble néanmoins jouer un rôle déterminant sur l'épaisseur de rubéfaction de la sole, comme nous le verrons pour le four de Sarcelles.

\section{LES QUESTIONS EN SUSPENS}

Le but recherché par les essais de chauffe et les expérimentations est donc de tenter d'individualiser les effets de chaque facteur sur la cuisson, de comprendre comment s'opère la rubéfaction pour enfin parvenir, à l'inverse, à identifier le mode d'utilisation d'une structure de combustion à partir de ses stigmates rubéfiés. Si l'étude des fours nous à permis d'émettre des hypothèses quant à l'utilisation, la longévité ou la place de cette structure au sein du village ; il reste à examiner de nombreux détails quant à leur mise en œuvre, fonctionnement et utilisation :

- Le temps passé au creusement de la cavité ou à l'élaboration d'une voûte construite.

- Quels outils sont utilisés ?

- Le choix entre voûte construite et voûte creusée n'est-il dicté que par la nature de l'encaissant?

- La mise en place de la rubéfaction, a-t-elle un rôle dans la fonctionnalité du four ? y a t'il des cuissons de préparation?

- Pourra t-on un jour déduire de l'épaisseur et/ou de la couleur de la sole une température ? et préciser les températures atteintes?

- Qu'elle est la durée de chauffe préparatoire à une cuisson ? Y a-t-il des cuissons où l'on garde la braise ? Cherchaient-ils à bien répartir la chaleur?

- Qu'elle est la durée de vie de ce type de structure ? 5, 10, 25 ans ou plus?

- Pourquoi certaines périodes sont marquées par la réfection des soles et d'autres par la mise en œuvre de fours nombreux?

- Les fours hémisphériques s'apparentent aux fours à pain, mais d'autres structures de combustion offrent le même type de rubéfaction. Sont-ils tous culinaires ? Y a-t'il des utilisations spécifiques?

- Un foyer peut-il offrir une sole comparable à celle des fours ? même croûte, même épaisseur?

Certaines réponses pourront venir de la découverte de nouveaux fours et d'autres seulement par la mise en œuvre d'essais de cuisson.

\section{DES ESSAIS DE CUISSONS PLUS QU'UNE APPROCHE EXPERIMENTALE}

Tous les essais qui ont été effectués pour l'heure ont été limités dans le temps car ils étaient le plus souvent réalisés dans le cadre de fouilles œuvre sont les plus aisées à reconstituer, les chauffes et la fonctionnalité des fours hémisphériques le sont moins. Ces cuissons ont souvent été l'occasion d'illustrer des journées portes ouvertes et se sont effectuées dans un cadre festif, cependant un souci expérimental a toujours été conservé en prenant note des dimensions, des temps de réalisation, de chauffe, d'épaisseur et dans la mesure du possible des températures de cuisson (fig. 3).

Pour ce qui concerne la mise en œuvre, la technique du four creusé est plus rapide que celle $\mathrm{du}$ four construit si le limon encaissant n'est pas trop sec. La construction de voûte nécessite un transport de matériaux, pierres, limon et bois. À Orville, l'association pierres et limon avait été préférée au torchis afin de coller aux découvertes observées lors de la fouille du site haut Moyen Âge voisin (GENTILI 2001). Le creusement nécessite uniquement un outil adapté ; le plus difficile étant d'obtenir une chambre de grande dimension. Le manche de l'outil doit être suffisamment long si l'on veut atteindre une profondeur de plus d'un mètre. Pour pallier le long manche, il est aussi possible d'entrer dans la chambre et de creuser par l'intérieur.

La première chauffe semble assécher le four ; une épaisse fumée se dégage lié à l'évaporation de l'humidité du limon encaissant. Il faudra plusieurs chauffes pour que la rubéfaction s'opère.

Même après plusieurs feux, le foyer ne peut être placé tout de suite au centre de la cavité car il manque d'air, il est allumé dans un premier temps sur le devant du four. Un apport de combustible en petit module permet de garder de bonnes flammes en cas de manque d'oxygène. Les conduits d'évent sont difficiles à mettre en œuvre (pour les fours creusés) et ne résolvent pas tous les problèmes de circulation d'air.

Les températures atteintes ont parfois été mesurées à l'aide d'une sonde placée à différents endroits du foyer, dans les braises et dans la cavité

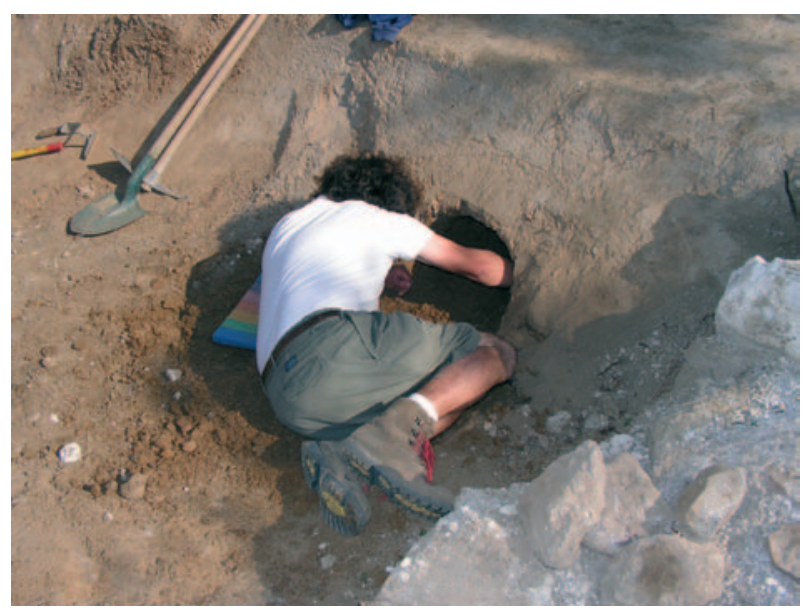

Fig. 3 - Vue du creusement. 


\begin{tabular}{|c|c|c|c|c|c|}
\hline & Villiers 1996 & Orville 2001 & Jossigny 2007 & Sarcelles 207 & Mauregard 2008 \\
\hline Nature encaissant & $\begin{array}{l}\text { limon sablo } \\
\text { argileux humide }\end{array}$ & calcaire & limon argileux & limon sableux & $\begin{array}{l}\text { limon sablo- } \\
\text { argileux humide }\end{array}$ \\
\hline $\begin{array}{l}\text { Temps de } \\
\text { creusement }\end{array}$ & $4 \mathrm{~h} 30$ & $\begin{array}{l}\text { four construit et } \\
\text { reconstruit. } 2 \mathrm{j}\end{array}$ & $5 \mathrm{~h}$ & 3 à $4 \mathrm{~h}$ & $3 \mathrm{~h} 30$ \\
\hline Outils & $\begin{array}{l}\text { truelle à } \\
\text { l'intérieur, binette }\end{array}$ & $\begin{array}{l}\text { truelle, pelle, } \\
\text { pierrre et limo, }\end{array}$ & truelle et rasette & truelle et rasette & $\begin{array}{l}\text { pelle extérieur, } \\
\text { truelle }\end{array}$ \\
\hline Diamètre & $\begin{array}{l}110 \text { (moyenne } \\
\text { archéologique) }\end{array}$ & 90 & & $70 * 80$ & 120 \\
\hline Sole & plane & $\begin{array}{l}\text { plane, radier de } \\
\text { pierre }\end{array}$ & plane & plane & plane \\
\hline Hauteur voûte & 50 & & & 29 & 40 \\
\hline Largeur bouche & 40 & & & 40 & 40 \\
\hline Hauteur bouche & 60 & & & 24 & $35 ?$ \\
\hline Cheminée & oui & non & non & non & non/oui \\
\hline Aménagement & $\begin{array}{l}\text { piédroits } \\
\text { calacaire puis } \\
\text { grès }\end{array}$ & $\begin{array}{l}\text { piedroits } \\
\text { apparents } \\
\text { voûte en pierres } \\
\text { recouverte } \\
\text { interne/ externe } \\
\text { de limon }\end{array}$ & $\begin{array}{l}\text { piedroits et } \\
\text { linteau grès }\end{array}$ & aucun & aucun \\
\hline Nombre chauffes & $\begin{array}{l}2 \text { dont } 1 \text { d'une } \\
\text { journée }\end{array}$ & $\begin{array}{l}\text { utilisé sur le long } \\
\text { terme }\end{array}$ & $\begin{array}{l}\text { 6, en demi- } \\
\text { journée }\end{array}$ & $\begin{array}{l}4 \text { longues (1 } \\
\text { journée) mais peu } \\
\text { intenses }\end{array}$ & 3 de $2 \mathrm{~h}$ chacune \\
\hline Type de cuisson & $\begin{array}{l}\text { chauffe ouverte } \\
\text { et fermée : tartes } \\
\text { et pain réussis } \\
\text { après } 1 \text { journée } \\
\text { de chauffe }\end{array}$ & $\begin{array}{l}\text { chauffe ouverte } \\
\text { et fermée : tartes, } \\
\text { pain }\end{array}$ & $\begin{array}{l}\text { chauffe ouverte } \\
\text { et fermée : pizza, } \\
\text { pain }\end{array}$ & chauffe ouverte & $\begin{array}{l}\text { chauffe ouverte } \\
\text { et fermée : pizza, } \\
\text { échec }\end{array}$ \\
\hline Combustible & $\begin{array}{l}\text { bûches, } \\
\text { branchages verts, } \\
\text { cagettes }\end{array}$ & $\begin{array}{l}\text { bûches, } \\
\text { branchages verts, } \\
\text { cagettes }\end{array}$ & & branchage, bûche & $\begin{array}{l}\text { bûchettes, } \\
\text { brindilles, } \\
1 \text { caisse }\end{array}$ \\
\hline Fermeture & pierre, limon & pierre, limon & pierre et limon & aucun & porte en bois \\
\hline Température foyer & $\begin{array}{l}850^{\circ}, 420^{\circ} \\
\text { ambiante }\end{array}$ & non mesurée & non mesurée & $410^{\circ}$ dans la sole & $\begin{array}{l}600^{\circ}, 250^{\circ} \\
\text { ambiante }\end{array}$ \\
\hline $\begin{array}{l}\text { Température } \\
\text { enfournement }\end{array}$ & $250^{\circ}$ puis chute & non mesurée & non mesurée & & $180^{\circ}$ puis chute \\
\hline $\begin{array}{l}\text { Prélèvement } \\
\text { magnétique }\end{array}$ & non & non & $\begin{array}{l}\text { oui, } 2008 \text { après } 1 \\
\text { an d'abandon }\end{array}$ & & impossible \\
\hline $\begin{array}{l}\text { Éaisseur } \\
\text { rubéfaction : voûte }\end{array}$ & non testé & non testé & & $\begin{array}{l}3 \text { à } 4 \mathrm{~cm} \text { base, } \\
\text { sommet } 6 \text { à } 7 \mathrm{~cm}\end{array}$ & 1 \\
\hline $\begin{array}{l}\text { Éaisseur } \\
\text { rubéfaction : sole }\end{array}$ & non testé & non testé & & $\begin{array}{l}3 \text { à } 6 \mathrm{~cm}(\mathrm{au} \\
\text { centre) }\end{array}$ & $\begin{array}{l}\text { en cours : } 1 \text { à } \\
2,5 \mathrm{~cm} \text { (centre et } \\
\text { sur le devant) }\end{array}$ \\
\hline
\end{tabular}

Tab. I - Tableau récapitulatif
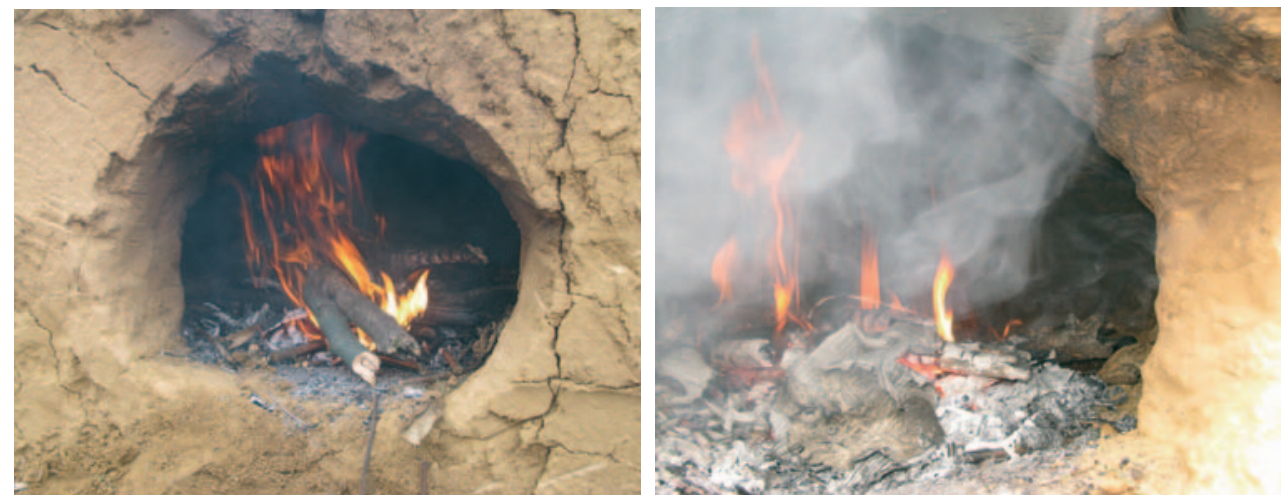

Fig. 4 - Foyer au cœur du four. 


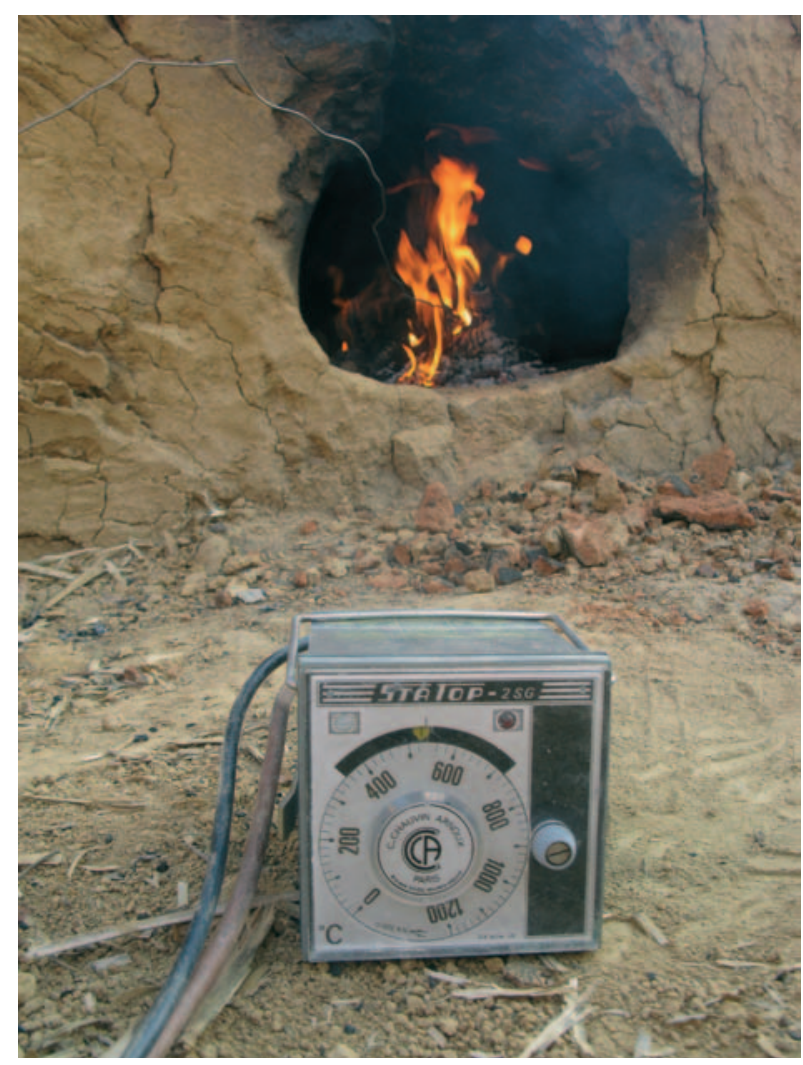

Fig 5 - Mesure de température.

avec les aliments seuls. Si dans le premier cas, les températures montent facilement autour de 600$800^{\circ}$, la répartition de la chaleur avec les braises décroît autour de $400^{\circ}$ et la chute s'accélère lorsque le foyer est retiré et le pain placé à l'intérieur. Plus la chambre de chauffe est grande, plus la tenue de la température est difficile.

Lors des études d'épaisseurs de rubéfaction, où le centre est toujours plus cuit que la périphérie, il nous a semblé plus pertinent de penser que le foyer était évacué pour la cuisson et non repoussé sur le pourtour. Pour procéder à cette cuisson fermée, la bouche est obturée au moyen d'une pierre, colmatée avec du limon ou d'une porte en bois.

Les cuissons ouvertes de pizza sont facilement réalisables alors que les cuissons fermées donnent des résultats plus aléatoires car exposées à la décroissance rapide de la chaleur. C'est sur ce point, qu'il nous apparaît nécessaire de tester sur le long terme les fours afin d'évaluer le meilleur temps de préchauffe et d'étudier l'impact de l'épaisseur de rubéfaction sur ces propriétés de conduction et de rayonnement.
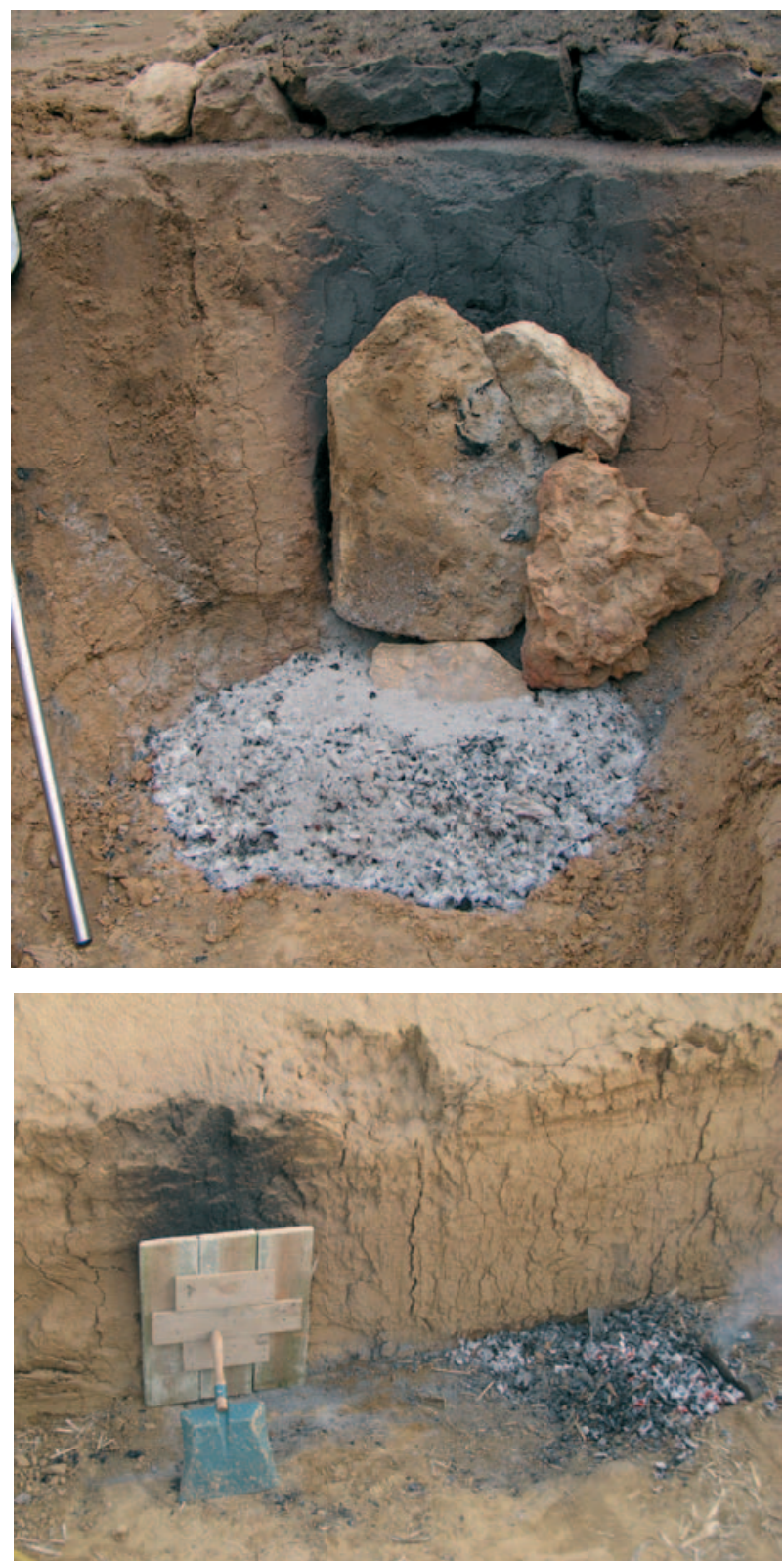

Fig. 6 - Exemple de porte en pierre (haut), exemple de porte en bois (bas).

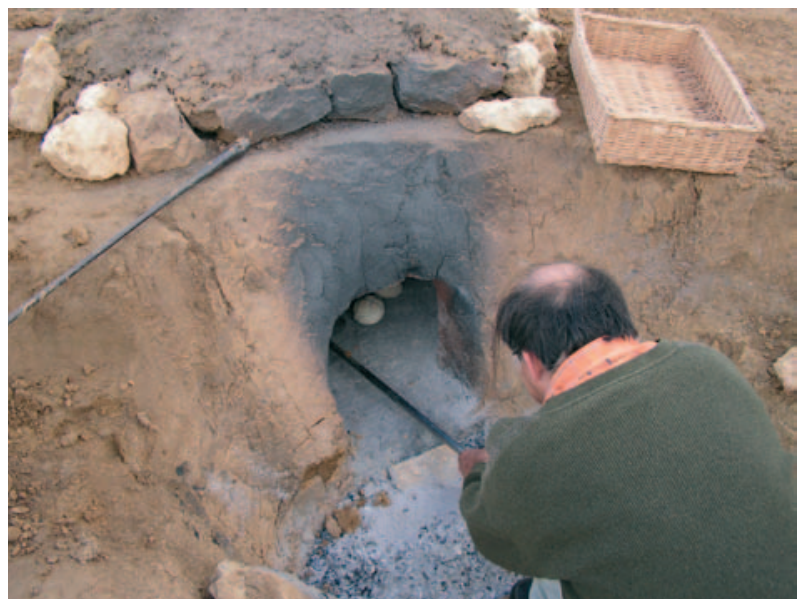

Fig. 7 - Cuisson du pain à Jossigny 


\section{RÉFLEXION SUR LA RUBÉFACTION : L'ANALYSE MAGNÉTIQUE DES FOURS EXPERIMENTAUX}

La construction et l'utilisation de fours culinaires expérimentaux ont été l'occasion d'étudier leurs propriétés magnétiques et de les comparer à celles des fours archéologiques.

En effet, de nombreuses études archéomagnétiques ont été menées en Île-de-France à partir de l'échantillonnage de fours culinaires mis au jour sur différents sites du haut Moyen Âge (WARMÉ 2009). Ces analyses ont permis d'avoir une bonne vision des caractéristiques magnétiques générales de ce type de structure. Il nous a alors semblé intéressant de vérifier si les caractéristiques magnétiques des fours expérimentaux étaient similaires. Nous avons pour ce faire pris soin de comparer les résultats expérimentaux avec les résultats archéomagnétiques obtenus sur des fours du même site (comparaison sur la base du même substrat).

Deux constatations ont pu être faites de ces études : l'une concerne le rapport entre le taux de viscosité et la température atteinte de la sole ; l'autre rend compte de l'effet des perturbations mécaniques subies par la sole après enfouissement sur la direction archéomagnétique enregistrée.

Il s'agit là bien entendu de premiers résultats tirés de deux études réalisées sur les fours expérimentaux de Jossigny et Sarcelles et qu'il conviendra de conforter par d'autres études.

\section{LE TAUX DE VISCOSITE ET LES TEMPERATURES DE CHAUFFE}

Les structures ou objets archéologiques constitués d'argile et qui ont subi une cuisson au moment de leur fabrication ou de leur utilisation peuvent acquérir une aimantation rémanente stable dans le temps (appelée aimantation thermo-rémanente) caractéristique du champ magnétique ambiant au moment de leur cuisson (la température requise pour une aimantation forte et stable est d'au moins $670^{\circ} \mathrm{C}$ ). Les oxydes de fer contenus dans les argiles peuvent en effet garder en mémoire la direction et l'intensité du champ magnétique ancien. Dans le cas des structures non déplacées après leur dernière chauffe, comme les fours culinaires, il est possible de retrouver la direction archéomagnétique enregistrée.

À l'aimantation thermo-rémanente se superpose en général une aimantation rémanente visqueuse qui est dûe au fait que certains grains magnétiques préalablement bloqués suivant une direction magnétique ancienne, tendent à se réaligner suivant la direction du champ magnétique ambiant.
Ce phénomène est, en première approximation, d'autant plus important que la structure n'a pas suffisamment chauffée (température atteinte inférieure à $670^{\circ} \mathrm{C}$ ).

Les fours culinaires archéologiques montrent des taux de viscosité moyens de l'ordre de 6 à $8 \%$ de l'aimantation totale (fig. 9). A partir de cette base il est possible, dans le cadre expérimental, de savoir si la sole a suffisamment monté en température. Les résultats obtenus sur les fours expérimentaux de Sarcelles et Jossigny en est une illustration : alors que le taux de viscosité du four de Jossigny est similaire au four archéologique 1038, le taux de viscosité élevé du four de Sarcelles traduit une température insuffisante de la sole (la température interne de la sole a été mesurée lors des chauffes et celle-ci ne dépassait effectivement pas $410^{\circ} \mathrm{C}$ ).

$\begin{array}{ll}\text { Jossigny expérimental } & 8 \% \\ \text { Jossigny F1038 } & 8.7 \% \\ \text { Sarcelles expérimental } & 13.7 \% \\ \text { Sarcelles F1106 } & 7.4 \%\end{array}$

Tab. II - Les viscosités moyennes des fours expérimentaux comparées à celle des fours archéologiques.

\section{L'EFFET DES PERTURBATIONS MÉCANIQUES SUR LA DIRECTION ARCHÉOMAGNÉTIQUE}

Durant leur enfouissement, les soles subissent de nombreuses bioturbations et autres perturbations mécaniques : la coupe de la sole expérimentale est bien homogène alors que celle de la sole archéologique F1038 comprend de nombreuses bioturbations (fig. 8).

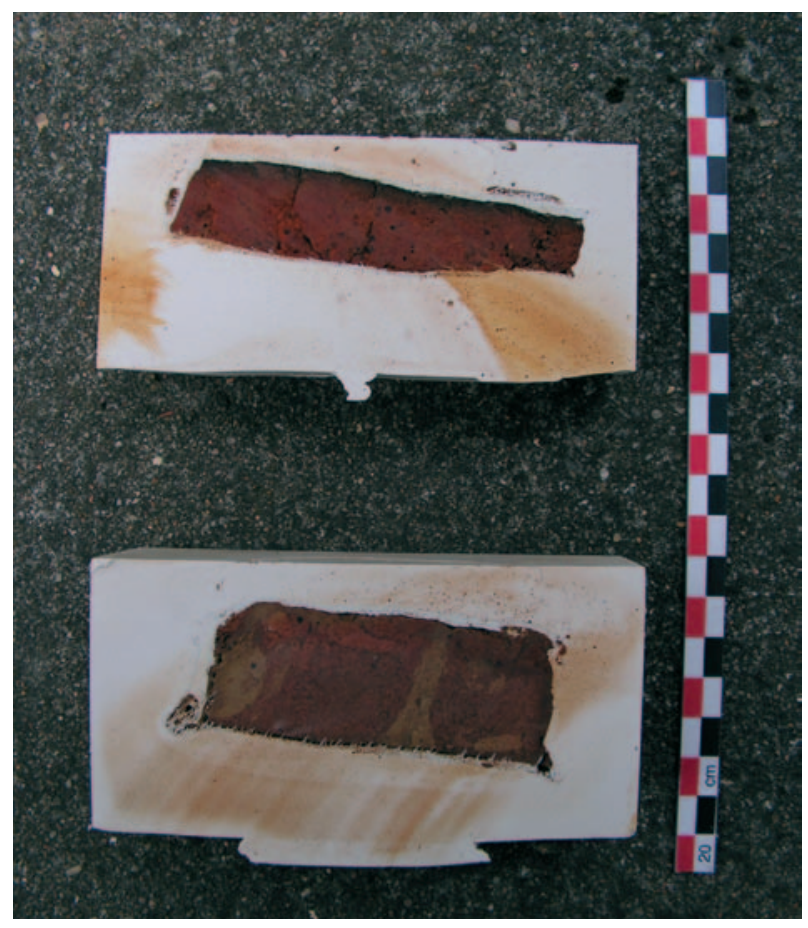

Fig 8 - Vue en coupe de la sole expérimentale (en haut) et de la sole archéologique (en bas) 


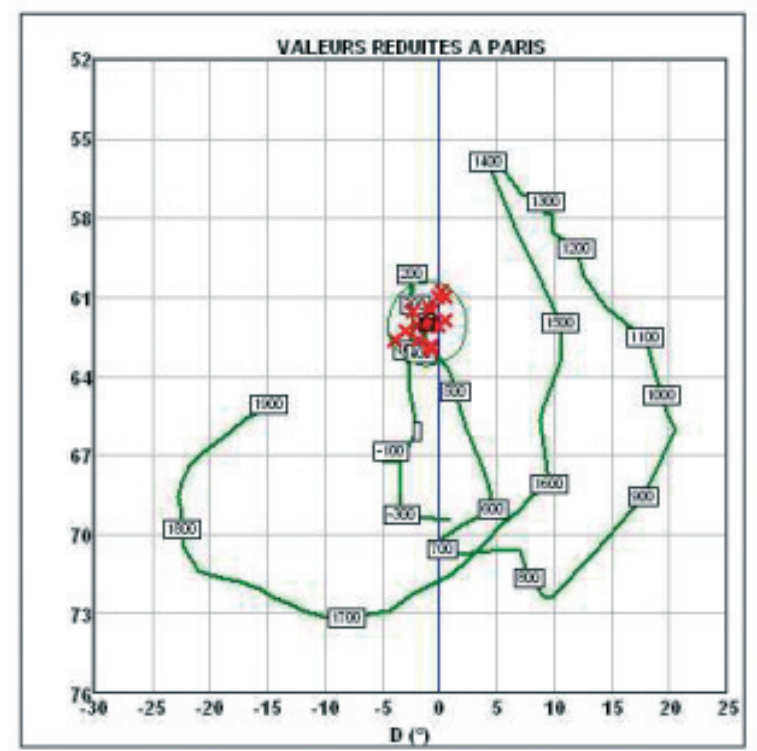

Fig 9 - Directions magnétiques individuelles des échantillons de la sole expérimentale.

Ces dernières entraînent une dispersion des directions magnétiques comme en témoigne les résultats obtenus sur les fours de Jossigny. La figure 9 représente les directions magnétiques individuelles des échantillons de chacun des fours (figurées par une croix rouge) ainsi que chacune des directions moyennes avec son incertitude à $95 \%$ (carré rouge associé à l'ovale de confiance) de l'ATR.

Dans le cas du four expérimental, les directions magnétiques individuelles sont très bien groupées (fig. 9). Il en résulte une direction moyenne très bien définie.

La sole du four archéologique F1038 montre des directions plus dispersées (fig. 9). Il en résulte une incertitude à $95 \%$ sur la direction moyenne plus grande ; l'espace de confiance est plus grand.

\section{PERSPECTIVES EXPERIMENTALES ET ETHNOGRAPHIQUES}

Une des voies que nous avons pour l'heure négligée est l'ethnographie. Après des conversations fructueuses avec un ressortissant de Moldavie qui connaissait et utilisait ce type de fours, il nous est apparu indispensable d'organiser, à moyen terme, une expédition d'observation et $\mathrm{d}$ 'apprentissage en Moldavie ou en Roumanie. Il semble que plusieurs types de fours creusés soient utilisés : leur morphologie variant en fonction de la préparation (pain, fumage de saucisses etc.). Ces différences pourront être comparées avec celles observées sur les sites du haut Moyen Âge qui livrent en effet plusieurs types de structures de combustion à vocation culinaire (HURARD \& BRULEY à paraître) : si leurs différences morphologiques sont évidentes, leurs particularités d'utilisation nous échappent encore.

En parallèle, nous souhaiterions approfondir notre approche expérimentale pour qu' elle devienne plus systématique et plus rigoureuse, pour cela quelques contraintes devront être surmontées :

- Trouver un lieu durable et approprié où le substrat est limoneux et où il serait possible de tester les fours sur plusieurs années. L'espace devra être suffisamment grand pour creuser plusieurs types de fours.

- Assurer un approvisionnement en bois et réflexion sur le combustible : selon la taille des fours à tester, une collecte simple peut s'avérer insuffisante et il faudra réfléchir à un financement.

- Le lieu devra être équipé d'une source électrique et du matériel de mesure afin de tester les températures de chauffe. Il faudrait investir dans des capteurs qui puissent rester dans les soles.

- L'élaboration d'un protocole reproductible est en partie réalisée en reprenant le tableau précédemment exposé ( $c f$. tab. I).

- La priseen compte des sources ethnographiques afin d'essayer les pratiques observées et de les comparer aux données archéologiques.

Les perspectives d'études, sur ce thème restent donc vastes et ouvertes à de nombreux approfondissements : les différentes pistes devront continuer à être abordées conjointement afin d'être confrontées. Cet article est aussi l'occasion de se faire connaître et d'inviter les personnes effectuant des essais de cuissons similaires à faire partager leurs expériences. 


\section{BIBLIOGRAPHIE}

BRULEY-CHABOT Gaëlle (2007) - « L'évolution des fours à pain entre le $\mathrm{IV}^{\mathrm{e}}$ et le $\mathrm{XII}{ }^{\mathrm{e}}$ siècle » dans La Neustrie $d u$ $V^{e}$ au $X^{e}$ siècle, Actes du XXVe colloque de l'AFAM 2004 à Tournai.

BRULEY-CHABOT Gaëlle (2007) - « Les fours et foyers culinaires du Haut Moyen Âge ", actes du colloque Medieval Europe Paris, disponible sur internet:

http://medieval-europe-paris-2007.univ-paris1.fr/G.BruleyChabot\%20poster.pdf

http://medieval-europe-paris-2007.univ-paris1.fr/G.BruleyChabot\%20texte.pdf
GENTILI François \& BRULEY-CHABOT Gaëlle (2000) Louvres "Le Bois d'Orville " : habitat rural du Haut Moyen $\hat{A} g e$, DFS de sauvetage urgent, Saint-Denis.

HURARD Séverine \& BRULEY-CHABOT Gaëlle à paraître - Saint-Pathus "Les Petits Ormes", rapport d'Opération, Inrap, SRA, à paraître.

WARME Nicolas, 2009 - « L'archéomagnétisme appliqué aux fours culinaires du Haut Moyen Âge : 10 ans d'activité en collaboration avec le PCR "Habitat rural du haut Moyen Âge" "dans L'habitat rural du Haut Moyen Âge en Ile-de-France, PCR bilan 2003/2005, suppl. au Bulletin archéologique du Vexin français, Guiry-en-Vexin, à paraître.

\section{Les auteurs}

Gaëlle BRULEY-CHABOT, Inrap

311 rue de Beauvais

F - 60300 Senlis

Nicolas WARMÉ, Inrap

42 rue de la Roche

F - 95300 Pontoise

\section{Résumé}

Les indices mis au jour par l'archéologie, concernant les fours du haut Moyen Âge, ont permis d'observer les variations morphologiques ainsi que les évolutions de répartition spatiale mais de nombreuses questions, propres à leur fonctionnement et utilisation, restent en suspens.

Les expérimentations menées, depuis plusieurs années, tentent de détailler, d'individualiser les facteurs qui agissent lors des cuissons : la rubéfaction du limon est la donnée essentielle qui relie le four archéologique au four expérimental. Les essais en sont encore à leur balbutiement, mais un protocole d'observation a été posé afin de comparer les expériences. En parallèle, des analyses magnétiques ont été menées afin d'évaluer l'impact de la morphologie et du sédiment entre autres, sur l'acquisition de l'aimantation dite thermo-rémanente (ATR) par les soles rubéfiées en place.

Mots-clés : expérimentation, fours, archéomagnétisme, haut Moyen Âge. 
RAP - 2009, n 1/2, Gaëlle BRULEY-С HABOT \& Nicolas WARMÉ, Approche expérimentale pour la compréhension des fours culinaires du haut Moyen Âge : bilan critique et perspectives. 